\title{
Two-Dimensional Electrons in a Strong Magnetic Field with Disorder: Divergence of the Localization Length
}

\author{
K. Ziegler \\ Institut für Theorie der Kondensierten Materie, Universität Karlsruhe, Physikhochhaus, D-76128 \\ Karlsruhe, Germany
}

(February 5, 1995)

\begin{abstract}
Electrons on a square lattice with half a flux quantum per plaquette are considered. An effective description for the current loops is given by a twodimensional Dirac theory with random mass. It is shown that the conductivity and the localization length can be calculated from a product of Dirac Green's functions with the same frequency. This implies that the delocalization of electrons in a magnetic field is due to a critical point in a phase with a spontaneously broken discrete symmetry. The estimation of the localization length is performed for a generalized model with $N$ fermion levels using a $1 / N$-expansion and the Schwarz inequality. An argument for the existence of two Hall transition points is given in terms of percolation theory.
\end{abstract}

PACS numbers: 71.55J, 73.20D, 73.20J

Typeset using REVTEX 
Lattice models for the two-dimensional electron gas in a strong magnetic field are of increasing interest because of recent experiments with GaAs heterostructures [1]. Moreover, lattice models offer new ways to the study of the quantum Hall transition. The purpose of this article is to discuss a new concept for the investigation of the localization length for the lattice model.

The tight-binding Hamiltonian for non-interacting electrons on a square lattice with magnetic flux $\phi$ reads in Landau gauge

$$
H=-t \sum_{r}\left[e^{2 i \pi y \phi / \phi_{0}} c^{+}\left(r+e_{x}\right) c(r)+c^{+}\left(r+e_{y}\right) c(r)+h . c .\right]+\sum_{r} V(r) c^{+}(r) c(r) .
$$

$e_{x, y}$ are lattice unit vectors, and $c^{+}$and $c$ are fermion creation and annihilation operators, respectively. $V(r)$ is a potential which represents an additional structure or disorder on the lattice. The dispersion for $V=0$ and half a flux quantum per plaquette $\left(\phi=\phi_{0} / 2\right)$ is $E= \pm 2 t \sqrt{\cos ^{2} k_{1}+\cos ^{2} k_{2}}$. A linear (relativistic) approximation for $E \sim 0$ around the four nodes $k_{j}= \pm \pi / 2$ is possible. For $H$ this is still very complicated [2], and a further simplification is useful to lift the degeneracy of the four nodes. This can be achieved by the introduction of a next nearest neighbor hopping term and a staggered potential in $H$ [3: 4 . This modification is motivated by the network model [5]. Expansion of $k=(\pi / 2, \pi / 2)+p$ for small $p$ vectors leads to the large scale approximation by the Dirac Hamiltonian $H^{\prime}=$ $\sigma \cdot p+\sigma_{3} M$ with Pauli matrices $\sigma_{j}$. Disorder, originally introduced in $H$ by a staggered random potential $V$, is described in $H^{\prime}$ by a random mass $M$ [3]. Physical quantities can be obtained from the Green's function

$$
G(\omega) \equiv\left(\begin{array}{ll}
G_{11} & G_{12} \\
G_{21} & G_{22}
\end{array}\right)=\left(\begin{array}{cc}
i \omega+M & h \\
h^{+} & i \omega-M
\end{array}\right)^{-1},
$$

where the Fourier component of $h$ is $p_{1}-i p_{2}$. For instance, the frequency-dependent conductivity is given by Kubo's formula

$$
\sigma_{x x}(\omega)=\frac{e^{2}}{h} \omega^{2} \sum_{r} r^{2}\left\langle G_{j j^{\prime}}(r, 0 ; i \omega) G_{j^{\prime} j}(0, r ;-i \omega)\right\rangle,
$$

where $G_{j j^{\prime}}(r, 0 ; i \omega) G_{j^{\prime} j}(0, r ;-i \omega)=\left|G_{j j^{\prime}}(r, 0 ; i \omega)\right|^{2}$. The localization length $\xi_{l}$ is defined as the decay of the function $C_{j j^{\prime}}(r, \omega) \equiv\left\langle\left|G_{j j^{\prime}}(r, 0 ; i \omega)\right|^{2}\right\rangle$ in space. There exists a relation of 
the above expression, which is composed of Green's functions at frequencies with opposite sign (retarded and advanced Green's functions), with a product of Green's functions at the same frequency. This follows from the block matrices in (2)

$$
G_{j j}\left(r, r^{\prime} ;-i \omega\right)=-G_{j^{\prime} j^{\prime}}\left(r^{\prime}, r ; i \omega\right), \quad G_{j j^{\prime}}\left(r, r^{\prime} ;-i \omega\right)=-G_{j j^{\prime}}\left(r^{\prime}, r ; i \omega\right) \quad\left(j \neq j^{\prime}\right) .
$$

This identity reflects the Lorentz-covariance of the Dirac theory. It implies $\left|G_{j j}\left(r, r^{\prime} ; i \omega\right)\right|^{2}=$ $-G_{j j}\left(r, r^{\prime} ; i \omega\right) G_{j^{\prime} j^{\prime}}\left(r, r^{\prime} ; i \omega\right)$ and $\left|G_{j j^{\prime}}\left(r, r^{\prime} ; i \omega\right)\right|^{2}=-G_{j^{\prime} j}\left(r, r^{\prime} ; i \omega\right) G_{j j^{\prime}}\left(r, r^{\prime} ; i \omega\right)$. That means only the Green's functions with one frequency is required for the evaluation of transport or localization properties in the present model.

The physics of the electrons can be understood as the statistics of current loops, created by the magnetic field. Depending on the potential there are local current loops with two different directions. This observation is central for the understanding of the Hall transition discussed subsequently. The current loops are composed from the Dirac fermions by the creation and annihilation of particle-hole pairs. The direction can be reversed globally by a timereversal transformation. In terms of the Green's function this means $G(\omega) \rightarrow-\sigma_{3} G(\omega) \sigma_{3}$. In general the system is not invariant under this transformation because one direction of the currents is favored. The favored direction characterizes the electronic state of a Hall plateau. The transition between the Hall plateaux corresponds to a symmetric point with $\omega=M=0$ which is also a critical point with a divergent localization length. The discrete symmetry at this critical point plays a fundamental role for the lattice electrons in a magnetic field. Taking now a random mass, which is symmetrically distributed around zero, this symmetry is spontaneously broken for the average Green's function [6]. It will be discussed in the following that the localization length also diverges at two critical points in the presence of disorder where the symmetry is broken. This is an extension of a previous work [7] where the Hall conductivity was calculated. Critical points with a divergent localization length in a symmetry broken regime are known from Anderson localization in systems without magnetic field and dimensionality $d>2$. However, the physics of the latter is different because there are no current loops. As a manifestation of this difference a continuous symmetry appears [8] 
instead of discrete symmetry found for localization in the presence of a magnetic field. The large scale approximation for the continuous symmetry leads to a non-linear sigma model contrary to the Dirac theory in the presence of the magnetic field.

The Schwarz inequality can be applied to get a lower bound for $C$ :

$$
\left|\left\langle G_{j j^{\prime \prime}}(r, 0 ; i \omega) G_{j^{\prime} j^{\prime \prime \prime}}(0, r ; i \omega)\right\rangle\right|^{2} \leq\left\langle\left|G_{j j^{\prime \prime}}(r, 0 ; i \omega)\right|^{2}\right\rangle\left\langle\left|G_{j^{\prime} j^{\prime \prime \prime}}(0, r ; i \omega)\right|^{2}\right\rangle
$$

with $j^{\prime \prime}=j, j^{\prime \prime \prime}=j^{\prime}$ or $j^{\prime \prime}=j^{\prime}, j^{\prime \prime \prime}=j$. Writing $C_{j j}^{\prime}(r, \omega) \equiv\left\langle G_{j j}(r, 0 ; i \omega) G_{j^{\prime} j^{\prime}}(0, r, i \omega)\right\rangle$ and $C_{j j^{\prime}}^{\prime}(r, \omega) \equiv\left\langle G_{j j^{\prime}}(r, 0 ; i \omega) G_{j^{\prime} j}(0, r, i \omega)\right\rangle$ one has $\left|C_{j j^{\prime}}^{\prime}(r, \omega)\right| \leq\left|C_{j j^{\prime}}(r, \omega)\right| . \quad C^{\prime}$ will be used subsequently because it easier to calculate than $C$. The average correlation functions are translational invariant. Therefore, the corresponding Fourier components $\tilde{C}(k, \omega)$ can be used to calculate the localization length

$$
\left.\xi_{l} \propto \sqrt{-\frac{\nabla_{k}^{2} \tilde{C}(k, \omega)}{\tilde{C}(k, \omega)}}\right|_{k=\omega=0}
$$

A simple calculation for the pure system gives $\xi_{l}=|M|^{-1}$. To evaluate the correlation function $C^{\prime}(r, \omega)$ a generalization of the Hamiltonian $H^{\prime}$ is introduced which describes $N$ levels of fermions per site [7]: $H^{\alpha \alpha^{\prime}}=H_{0}^{\alpha \alpha^{\prime}}-\delta M_{r}^{\alpha \alpha^{\prime}} \sigma_{3}\left(\alpha, \alpha^{\prime}=1,2, \ldots, N\right)$ with $H_{0}^{\alpha \alpha^{\prime}}=$ $\left(\sigma \cdot p+\langle M\rangle \sigma_{3}\right) \delta^{\alpha \alpha^{\prime}}$. The distribution of the real symmetric random matrix (a Gaussian Orthogonal Ensemble [9]) $\delta M$ is given by $\left\langle\delta M_{r}^{\alpha \alpha^{\prime}} \delta M_{r^{\prime}}^{\alpha^{\prime \prime} \alpha^{\prime \prime \prime}}\right\rangle=(g / N) \delta^{\alpha \alpha^{\prime \prime \prime}} \delta^{\alpha^{\prime} \alpha^{\prime \prime}} \delta_{r, r^{\prime}}$. That means only random fluctuations couple the $N$ different Dirac systems. The product of two Green's functions with the same frequency can be expressed as a functional integral [10]

$$
G_{j, j^{\prime \prime}}^{\alpha \alpha^{\prime}}\left(r, r^{\prime} ; i \omega\right) G_{j^{\prime}, j^{\prime \prime \prime}}^{\alpha^{\prime \prime} \alpha^{\prime \prime \prime}}\left(r^{\prime \prime}, r^{\prime \prime \prime} ; i \omega\right)=-\int \bar{\Psi}_{r^{\prime}, j^{\prime \prime}}^{\alpha^{\prime}} \Psi_{r, j}^{\alpha} \bar{\chi}_{r^{\prime \prime \prime}, j^{\prime \prime \prime}}^{\alpha^{\prime \prime \prime}} \chi_{r^{\prime \prime}, j^{\prime}}^{\alpha^{\prime \prime}} \exp \left(-S_{1}\right) \prod_{r} d \Phi_{r} d \bar{\Phi}_{r}
$$

with the action (sum convention for the level index $\alpha$ )

$$
S_{1}=i \sigma_{\omega}\left[-\left(\Phi,\left(H_{0}+i \omega \sigma_{0}\right) \bar{\Phi}\right)+\sum_{r} \delta M_{r}^{\alpha \alpha^{\prime}}\left(\Phi_{r}^{\alpha^{\prime}} \cdot \sigma_{3} \bar{\Phi}_{r}^{\alpha}\right)\right]
$$

$\sigma_{\omega}=\operatorname{sign}(\omega)$ and the field is $\Phi_{r, j}^{\alpha}=\left(\Psi_{r, j}^{\alpha}, \chi_{r, j}^{\alpha}\right)$. The first component is Grassmann and the second is complex. The complex component is added to normalize the functional integral in (7). It also provides a transparent representation of the product of Green's functions 
with the same frequency as required for $C$ and $C^{\prime}$. Averaging with Gaussian distributed fluctuations yields $\left\langle\exp \left(-S_{1}\right)\right\rangle=\exp \left(-S_{2}\right)$ with

$$
S_{2}=-i \sigma_{\omega}\left(\Phi,\left(H_{0}+i \omega \sigma_{0}\right) \bar{\Phi}\right)+\frac{g}{N} \sum_{r}\left(\Phi_{r}^{\alpha} \cdot \sigma_{3} \bar{\Phi}_{r}^{\alpha^{\prime}}\right)\left(\Phi_{r}^{\alpha^{\prime}} \cdot \sigma_{3} \bar{\Phi}_{r}^{\alpha}\right)
$$

This action can be mapped onto another effective field theory [11]: The interaction in $S_{2}$ can also be created by complex $2 \times 2$-matrix fields $Q, P$ and a complex Grassmann field $\psi$ which couple to the composite field $\sum_{\alpha=1}^{N} \Phi_{r}^{\alpha} \bar{\Phi}_{r}^{\alpha}$. The new field does not depend on $\alpha$, the level index of the $N$ level fermions. The level degree of freedom can be eliminated by integrating out the field $\Phi$ in the functional integral. This leads to $\exp (-N S(Q, P, \psi))$ with the action [1]

$$
\begin{gathered}
S(Q, P, \psi)=\frac{1}{g} \sum_{r}\left[\operatorname{Tr}_{2}\left(Q_{r} \sigma_{3}\right)^{2}+\operatorname{Tr}_{2}\left(P_{r} \sigma_{3}\right)^{2}\right]+\log \operatorname{det}\left(H_{0}-2 Q+i \omega \sigma_{0}\right) \\
-\log \operatorname{det}\left(H_{0}+2 i P+i \omega \sigma_{0}\right)+\frac{1}{N} \sum_{\mu, \mu^{\prime}=1}^{4} \int(\mathbf{I})_{\mu, \mu^{\prime}}(k) \psi_{k, \mu} \bar{\psi}_{-k, \mu^{\prime}} d^{2} k+O\left(N^{-2}\right) .
\end{gathered}
$$

Since the number of fermion levels $N$ appears in front of the new action, the limit $N \rightarrow \infty$ corresponds to a saddle point integration for the fields $Q$ and $P$. $\mathbf{I}(k)$ is the matrix of the Gaussian fluctuations around the saddle point solution $Q=Q_{0}+\delta Q$ and $P=i Q_{0}+\delta P$, where $Q_{0}$ is the $N \rightarrow \infty$ solution $Q_{0}=-(1 / 2)\left[i \eta \sigma_{0}+M_{s} \sigma_{3}\right] . M_{s}$ is a shift of the average Dirac mass and $\eta$ shifts the frequency in the Green's function. Introducing $m=\langle M\rangle+M_{s}$ as the effective (renormalized) Dirac mass the imaginary shift is $\eta=\sigma_{\omega} \sqrt{e^{-2 \pi / g}-m^{2}}$ for $|m| \leq m_{c} \equiv e^{-\pi / g}$ and zero otherwise [11].

The details of the derivation of $S(Q, P, \psi)$ from $S_{2}$ and the evaluation of saddle point integration can be found in Ref. [11]. However, (10) can also be reconstructed using the fact that $\int \exp \left(-S_{1}\right) d \ldots=1$. The $N \rightarrow \infty$-limit gives $Q_{0}=-i P_{0}$ which implies that the large $N$-terms cancel each other in the action (10). First order fluctuations do not contribute at the saddle point. And second order fluctuations appear as quadratic forms of $\delta Q$ and $\delta P$ with the matrix I. Therefore, the condition $\int \exp (-N S(Q, P, \psi)) d \ldots=1$ is satisfied if a quadratic form of $\mathbf{I}$ appears with a complex Grassmann field. This result reflects the supersymmetric construction of the functional integral. 
For the asymptotic behavior of a large correlation length it is sufficient to consider $k \sim 0$. If $k=0$ the matrix $\mathbf{I}$ reads

$$
\mathbf{I}(k=0)=\left(\begin{array}{cccc}
1 / g-2 \alpha \mu^{2} & 0 & 0 & 2 \beta \\
0 & 2 / g+4 \alpha|\mu|^{2} & 0 & 0 \\
0 & 0 & 2 / g+4 \alpha|\mu|^{2} & 0 \\
2 \beta & 0 & 0 & 1 / g-2 \alpha \mu^{* 2}
\end{array}\right),
$$

where $\mu=m+i \eta, \alpha=\int\left(|\mu|^{2}+k^{2}\right)^{-2} d^{2} k / 4 \pi^{2}$ and $\beta=\int k^{2}\left(|\mu|^{2}+k^{2}\right)^{-2} d^{2} k / 4 \pi^{2}$. The inner $2 \times 2$ matrix of $\mathbf{I}(k=0)$ is diagonal with positive matrix elements $2 / g+4 \alpha|\mu|^{2}$. It leads always to a finite correlation length. However, this part of $\mathbf{I}$ does not contribute to $C^{\prime}$ because $C_{j j^{\prime}}^{\prime}=\mathbf{I}_{j+2(j-1), j^{\prime}+2\left(j^{\prime}-1\right)}^{-1}$ [1]. Therefore, only the projected submatrix ( $P_{14}$ is the projector on the indices 1,4$)$

$$
P_{14} \mathbf{I}^{-1} P_{14}=\left(\begin{array}{cc}
1 / g-2 \mu^{2} \alpha+\mu^{2} A_{1} k^{2} & 2 \beta+B_{1} k^{2} \\
2 \beta+B_{1} k^{2} & 1 / g-2 \mu^{* 2} \alpha+\mu^{* 2} A_{1} k^{2}
\end{array}\right)^{-1}
$$

with positive constants $A_{1}, B_{1}$ is of interest here. It describes the critical behavior of the model due to one vanishing eigenvalue for $k=0$ at two critical points $m= \pm m_{c}$. The critical points are also characterized by a vanishing imaginary part of the saddle point $Q_{0}$.

The Fourier components of $C^{\prime}$ are of the form $\tilde{C}^{\prime}(k, \omega=0)=\left(2 \beta+b k^{2}\right) /\left(a^{\prime}+b^{\prime} k^{2}\right)$, where $b$ and $b^{\prime}$ are finite constants and $a^{\prime}=\left|1 / g-2 \alpha \mu^{2}\right|^{2}-4 \beta^{2}$. Equ. (6) yields for the correlation length $\xi$ of $C^{\prime}$

$$
\xi \propto 2\left(b^{\prime} / a^{\prime}-b / 2 \beta\right)^{1 / 2} \sim 2 \sqrt{b^{\prime} / a^{\prime}} .
$$

Because $b^{\prime}$ is always finite the divergence of the correlation length is determined by $1 / a^{\prime}$. The behavior of $a^{\prime}$ distinguishes the regime of $|m| \leq e^{-\pi / g}$ and $|m|>e^{-\pi / g}$. This leads to

$$
\xi \sim \xi_{0}\left(1 / g+\frac{1}{\pi} \log |m|\right)^{-1 / 2} \text { for }|m|>e^{-\pi / g}
$$

and

$$
\xi \sim \xi_{0}^{\prime}\left(e^{-2 \pi / g}-m^{2}\right)^{-1 / 2} \text { for }|m| \leq e^{-\pi / g} .
$$

Thus $\xi(m)$ diverges linearly at $m= \pm e^{-\pi / g}$ (then $\eta=0$ according to the saddle point solution), and it is finite elsewhere. The existence of two critical points, instead of the single 
critical point in the perturbative approach of Ref. [3] is a consequence of the spontaneous symmetry breaking due to disorder which creates a non-vanishing density of states between the particle and hole band of the Dirac theory. This non-perturbative phenomenon was discussed in Ref. [6, [1, 11].

The correlation function $C$ cannot be calculated directly from $S(Q, P, \psi)$ because it not expressable as a simple form in the large $N$ limit. Therefore, only $C^{\prime}$ can be used as a lower bound for the decay of $C$ due to inequality (5). As a consequence, $\xi$ bounds the localization length $\xi_{l}$ from below. An alternative approach, based on a different effective field theory with $N=1$, indicates also a square root divergent localization length [12]. The exponent $\nu \approx 1$ for the localization length was found experimentally for the metal-insulator transitions (i.e. near the mobility edge) in AlGaAs/GaAs heterostructures [1] and in Si MOSFETs [13].

On the other hand, numerical results for network models and lowest Landau level approaches [5.14 16] suggest that the localization length $\xi_{l}$ diverges at the critical point with an exponent $\nu \approx 7 / 3$.

The existence of two transition points is in disagreement with the single transition point of other approaches to the integer Hall transition [5, 14 [17]. This is either due to different models (Landau level approach versus lattice fermions) or due to the fact that the two transition points were not seen because of limitations of the numerical resolution. (The distance of the two transitions is $2 e^{-\pi / g}$ which is extremely small for weak disorder. It can be hidden by finite size effects.) Two transition points are also supported by the percolation picture for the lattice model. This can be seen considering a lattice where the space-dependent Dirac mass $M_{r}$ is either zero with probability $p$ or $M$ with probability $1-p$. The correlation length $\xi$ is infinite for $p=1$ because $M=0$ is the symmetric point. For $p<1$ not all lattice sites have a zero mass. However, there is typically an infinite cluster of lattice sites with zero mass for $p_{c}<p<1$ according to percolation theory [18]. The correlation length of the classical percolation system is finite for $p<p_{c}$ and diverges like $\xi \sim \xi_{0}\left(p_{c}-p\right)^{\nu}$ with the critical exponent $\nu=4 / 3$. The mean value of $M_{r}$ at the critical point $p_{c}$ is $\langle M\rangle=\left(1-p_{c}\right) M \equiv M_{c}$. It seems plausible that its value is non-zero even if a renormalization $(\langle M\rangle \rightarrow m)$ by quan- 
tum fluctuations of the fermions is taken into account. Application of the transformation $G \rightarrow \sigma_{3} G \sigma_{3}$ to the Green's function $G(\omega=0)$ replaces $M$ by $-M$. Thus $-M_{c}$ is also a critical point.

In conclusion, it was shown that the transport of electrons on a square lattice in a strong magnetic field and random potential can be described by a product of Dirac Green's function with the same frequency. Therefore, the physics is characterized by a discrete symmetry. The localization length diverges at two different critical points. The critical exponent was estimated by $\nu \geq 1 / 2$. 


\section{REFERENCES}

[1] A.A.Shashkin, V.T.Dolgopolov, G.V.Kravchenko, M.Wendel, R.Schuster, J.P.Kotthaus, R.J.Haug, K. von Klitzing, K.Ploog, H.Nickel and W.Schlapp, Phys.Rev.Lett.73, 3141 (1994)

[2] M.P.A.Fisher and E.Fradkin, Nucl.Phys. B251 [FS13], 457 (1985)

[3] A.W.W.Ludwig, M.P.A.Fisher, R.Shankar and G.Grinstein, Phys. Rev. B 50, 7526 (1994)

[4] M.Oshikawa, Phys.Rev. B 50, 17357 (1994)

[5] J.T.Chalker and P.D.Coddington, J.Phys. C 21, 2665 (1988)

[6] K.Ziegler, Nucl.Phys.B285 [FS19], 606 (1987)

[7] K.Ziegler, Europhys.Lett.28, 49 (1994)

[8] F.Wegner, Z.Physik B38, 207 (1979)

[9] M.L.Mehta, Random matrices (Acad. Press New York, 1967)

[10] J.W.Negele and H.Orland, Quantum Many - Particle Systems (Addison - Wesley, New York, 1988)

[11] K.Ziegler, Nucl.Phys.B 344, 499 (1990); Europhys.Lett.14 , 415 (1991)

[12] K.Ziegler (unpublished)

[13] V.T.Dolgopolov, G.V.Kravchenko and A.A.Shashkin, Phys.Rev. B46, 13303 (1992); A.A.Shashkin, V.T.Dolgopolov and G.V.Kravchenko Phys.Rev. B49, 14486 (1994)

[14] B.Huckestein and B.Kramer, Phys.Rev.Lett. 64, 1437 (1990)

[15] B.Mieck, Europhys.Lett. 13, 453 (1990)

[16] Y.Huo and R.N.Bhatt, Phys.Rev.Lett. 68, 1375 (1992) 
[17] A.M.M. Pruisken, in The Quantum Hall Effect, edited by R.E.Prange and S.M.Girvin (Springer-Verlag, New York, 1990)

[18] W.Essam, in Phase Transitions and Critical Phenomena Vol. 2, edited by C.Domb and M.S.Green (Academic Press, London 1972) 